\title{
The beneficial effects of intermittent fasting: an update on mechanism, and the role of circadian rhythm and gut microbiota
}

\author{
Dandan $\mathrm{Hu}^{1 \#}$, Zhibo Xie ${ }^{2 \#}$, Yuqian $\mathrm{Ye}^{2}$, Suhad Bahijri ${ }^{3,4}$, Minshan Chen ${ }^{1}$ \\ ${ }^{1}$ Department of Liver Surgery, Sun Yat-Sen University Cancer Center, Guangzhou, China; ${ }^{2}$ Department of Surgery, Peking Union Medical College \\ Hospital, Beijing, China; ${ }^{3}$ Department of Clinical Biochemistry, Faculty of Medicine, King Abdulaziz University, Jeddah, Saudi Arabia; ${ }^{4}$ Saudi \\ Diabetes Study Research Group (SDRG)-King Fahd Medical Research Centre, King Abdulaziz University, Jeddah, Saudi Arabia \\ Contributions: (I) Conception and design: D Hu, M Chen; (II) Administrative support: M Chen; (III) Provision of study materials or patients: D Hu, \\ Z Xie; (IV) Collection and assembly of data: Z Xie; (V) Data analysis and interpretation: Y Ye; (VI) Manuscript writing: All authors; (VII) Final \\ approval of manuscript: All authors. \\ \#These authors contributed equally to this work. \\ Correspondence to: Minshan Chen. Department of Liver Surgery, Sun Yat-Sen University Cancer Center, No 651 Dongfeng East Road 510060 , \\ Guangzhou, China. Email: chenmsh@sysucc.org.cn.
}

\begin{abstract}
Importance: There is accumulating evidence that intermittent fasting (IF) is connected to improved health condition and longevity time-restricted feeding (TRF) is the most recognized and extensively studied model of IF.

Objective: To investigate the underlying mechanism of pleiotropic benefits of IF and hint the most advantageous feeding pattern for humans.

Evidence review: We searched MEDLINE, EMBASE, Cochrane Library and Google Scholar by 2020 April for publications on IF or TRF and their mechanisms. Studies include animal models and human cohorts.

Findings: One important mechanism is that IF allows certain period of fasting time, in which our bodies activate pathways of repair and rejuvenation. Moreover, the advantages of IF, especially TRF over total caloric restriction (CR) provided bases for various animal and human studies which suggested that the feeding-fasting rhythm stimulates the fluctuation of our gut microbiota and a series of subsequent molecular alterations, which in turn restored a healthier circadian clock that resembled our inherent clock formed throughout millions of years of homo sapiens history.
\end{abstract}

Conclusions and Relevance for Reviews: Complete understanding of the mechanism leading to the beneficial effects of IF paves the way for tailored dietary regimen to combat a wide range of diseases and ill health conditions.

Keywords: Intermittent fasting (IF); circadian rhythm; gut microbiota

Submitted Feb 22, 2020. Accepted for publication Jun 04, 2020.

doi: 10.21037/hbsn-20-317

View this article at: http://dx.doi.org/10.21037/hbsn-20-317

\section{Intermittent fasting (IF) improves health phenotypes}

Different patterns of IF have been extensively studied in human subjects and animal models, and proven to improve numerous health conditions including obesity, impaired glucose tolerance, dyslipidemia, hypertension, fertility problems, liver impairment, and neurodegenerative diseases (1-13). Time-restricted feeding (TRF), which entails restricting food intake to a $6-8 \mathrm{~h}$ time period daily, is one of the classical IF models. Our previous study, using an ischemic-reperfusion model in mice, showed that TRF is protective against liver impairment (14), and hence was proposed as a potential intervention to be integrated into 
the standard perioperative care. Furthermore, IF can be tolerated by humans for long periods, and at repeated intervals as noted in the Islamic practice of fasting during the month of Ramadan yearly by devout Moslems of different ethnicities.

On the other hand, caloric restriction (CR), another popular format of dietary regimens, which was reported in various studies to provide positive effects for the management of multiple unfavorable health issues $(1,2)$, is difficult to maintain in the long run, and hence impractical to use in the management of chronic conditions (8).

Therefore, we will review various animal and human studies covering various aspects of IF in an attempt to explain reasons and possible mechanisms for its reported benefits.

We present the following article in accordance with the Narrative Review reporting checklist (available at http:// dx.doi.org/10.21037/hbsn-20-317).

\section{IF is a more tolerable feeding pattern}

It appears that all sorts of IF are promising feeding alternatives for management of various conditions affecting health and wellbeing.

One of the reasons for the compatibility of IF with human feeding patterns can be deduced from studies of the history of human eating habits. Humans, like other animals, evolved in environments where food source was relatively scarce, and hence, they developed feeding-fasting cycles based on the food availability (12). Indeed, our huntergatherer ancestors were adapted to lack of food most of the time before the emergence of farming civilization. In China, according to historical records, the three meals a day in present day society was only practiced among royal families before Tang and Song Dynasty (a few hundreds of years ago). On the other hand, one or two meals a day were more common among ordinary people, which largely resembles TRF (7). Therefore, based on survival of the fittest theory, our ancestors were those people carrying genes which were well-adapted to the high level of physical activity associated with our hunter-gatherer existence, and able to survive in the presence of limited food supply, that is "thrifty genes". However, not much genetic evolution has occurred in humans during the past 20,000 years, but there has been more rapid socio-economic development, accompanied by the adoption of our present sedentary lifestyle in the presence of abundant food supply. Therefore, our "thrifty genotype", inherited from our ancestors, is no longer adapted to our present day lifestyle, and has become a 'susceptibility genotype' for obesity and metabolic dysregulation, apparent as increased prevalence of chronic diseases and conditions such as metabolic syndrome, type 2 diabetes, dyslipidaemia, hypertension, cardiovascular disease (CVD), and even some types of cancer.

\section{Fasting physiology triggered by food deprivation is a key factor, but not the only explanation for potential health benefits of IF}

Fasting enables organisms to enter alternative metabolic phases, which set the stage for improved metabolic traits and healthy lifespan in animals $(1,12)$. This biological process can be attributed to waste evacuation and restoration of a stable internal microenvironment (12). The concept of 'fasting physiology' refers to changes in cellular responses due to restriction of food intake, so that cells rely less on glucose and more on ketone body-like carbon as fuel sources, which is reported to improve glucose homeostasis, mitochondrial function, and DNA repair (13). In addition, it stimulates autophagy, stem cell renewal, stress resistance, and suppresses inflammation $(10,13)$. Furthermore, these metabolic changes are reported to consume fat, causing weight loss (15), as well as providing a more diverse gut flora (16). Furthermore, during fasting, defenses against oxidative and metabolic stress, and removal of damaged molecules are also enhanced (17). On the other hand, during feeding state, insulin-pAKT-mTOR pathway drives downstream activities that promote anabolic processes, while AMPK activated by fasting inhibits mTOR activity $(13,18)$.

However, most people do not benefit from the above mentioned fasting physiology, because consuming three or more meals a day plus snacks would prevent initiation of the food deprivation process $(19,20)$. In contrast, IF of any kind allows a period long enough for the occurrence of fasting state. In particular, TRF, which restricts the food intake within a few hours per day, triggers the fasting physiology after 16-18 hours of feeding cessation on a daily basis.

It has also been reported that caloric restriction (CR) and IF, in spite of inducing similar weight loss, have considerably different effects on glucose and lipid metabolism (8). This may indicate that feeding schedule plays a critical role in metabolic related traits. Indeed, results from clinical trials comparing CR (reducing 20-50\% of total energy intake) and intermittent fasting (IF) concluded that even though IF did not lead to as much weight loss as CR, it achieved similar effects on the reduction of visceral fat, insulin concentration and insulin resistance (8). Furthermore, the 
obtained data suggested that after 3-24 weeks of IF or CR, $3-8 \%$ and $4-14 \%$ reductions in body weight were observed, respectively. However, greater degrees of energy restriction produced larger reductions in body weight. On the other hand, insulin levels were found to be reduced by $20-31 \%$ after 8-12 weeks of IF, and by $11-41 \%$ after $6-12$ weeks of CR (8).

From the above it can be suggested that even though fasting physiology, and accompanying metabolic changes plays an important part in the positive impact of IF on health, the noted effects could not be entirely attributed to these changes.

\section{Resemblance of natural circadian clock provides additional benefits}

Circadian rhythm ensures adaptation to natural conditions, where as disturbance of this rhythm causes pleiotropic adverse effects, including weight gain, disruption of glucose and lipid metabolism, etc. Circadian rhythm is a $\sim 24 \mathrm{~h}$ physiological change regulated in two ways in humans: (I) by light via the suprachiasmatic nucleus (central), and (II) by clock proteins that are present in almost every cell (peripheral). Rhythms in mammals are sustained by cellautonomous interlocked transcription-translation feedback loops. In this bioprocess, the transcriptional activators BMAL1 and CLOCK or NPAS2 bind to Per1, Per2, Cry1, and Cry2 genes, there by driving their transcription. Following an increase in the protein levels of PER and CRY, the transcription of CLOCK/NPAS2/BMAL1 is repressed. Subsequent degradation of PER and CRY thus closes the loop (21). Similar interlocked loop functions through interactions between CLOCK and BMAL1, and ROR and the REV-ERB family of transcription factors (22-24).

Although the circadian oscillator is basically cell autonomous, environmental parameters including light/dark switch, feeding/fasting cycle, temperature, humidity, etc. have significant influence on it. Many circadian transcripts are also targeted by transcriptional regulators which are activated or inhibited by the above environmental factors (25-27). One perfect example of such an environmentinternal environment interaction is shown when a jetlag mouse model experienced loss of rhythmicity in gut microbiota, which resulted in weight gain and glucose intolerance (28). The study first used taxonomic analysis of fecal microbiota from wild-type mice fed normal chow every 6 hours to demonstrated that daily fluctuations of the gut microbiota were significant in more than $15 \%$ of all bacterial operational taxonomic units (OTUs). To test the correlation of circadian clock of the host and the rhythmicity of the gut microbiota, the researchers then proved that the fluctuations in the flora were nearcompletely dampened in Per1/2-/- mice (circadian rhythm deficient model). In addition, a jet-lag model was created in wild-type mice by subjecting the mice to an 8-hour light cycle advance for 3 days, then reverting back to the routine light-dark cycle for another 3 days. Irregular food intake pattern was observed in this jet-lagged model, as well as loss of diurnal rhythm in the gut micro-environment which resembled the Per1/2 -/- mice. Finally, to verify the role of gut microbiota in the increased susceptibility to metabolic disease induced by environment, fecal transplantation from the jet-lagged human samples into germ-free mice were performed. Control groups included samples from human subjects before experiencing jet lag, as well as after recovering from jet lag. As a result, the germ-free mice colonized with micro flora from jet-lagged individuals showed significant weight gain and higher blood glucose concentration after oral glucose challenge (28).

\section{Feeding pattern causes rhythmic changes in our bodies}

Another important environmental factor is related to food intake. The ingestion of food causes sudden rise of nutrients from a sufficiently low basal level, and induces acute expression of clock genes (29), which influence the circadian rhythm (30). Studies have shown that TRF, without reducing caloric intake, could improve cell functions and prevent metabolic diseases in mice fed a highfat diet (6). as well as lower the incidence of cancerous diseases, by modulating multiple biological pathways $(6,9)$. Further studies reported that the mTOR pathway, which is activated by eating, leads to the phosphorylation of casein kinase 1 (CK1), and glycogen synthase kinase 3 (GSK3), both of which alter the stability of the circadian clock component PER (31). On the other hand, fasting activates AMP dependent protein kinase (AMPK), which initiates phosphorylation of CRY and promotes its degradation (32). In Addition, the cellular levels of both nicotinamide adenine dinucleotide (NAD) and Sirtuins, which affect the circadian rhythm, fluctuate with the energy state in the cells (33-35). Therefore, feeding/fasting cycle enhance the amplitude of the oscillation of circadian activators and repressors, which in turn regulate rhythmic tissue-specific transcriptomes $(6,27)$, and finally translate into healthier phenotypes.

The above findings were further verified by an animal 
model study using circadian clock mutant mice (3). This study used whole body Cry1;Cry2 knockout (CDKO) mice, and liver-specific Bmal1 and liver-specific Rev-erb $\alpha / \beta$ knockout mice, and assigned them to either high fat regimen served ad libitum (FA) or time-restrictedly (TRF). These mice are usually more susceptible to metabolic diseases because they lack the mechanism to modulate normal metabolic homeostasis. After 12-week of feeding high-fat diet, Rev-erba/bLDKO mice on FA gained $79.5 \%$ weight compared with only $31.3 \%$ for mice on TRF. Same trends were observed but to a more moderate degree in the other two models. Furthermore, in TRF groups, reduced fat mass and increased lean mass was noted in Bmal1LKO mice, Rev-erba/bLDKO mice, and CDKO mice by differing degrees. Moreover, the respective wild-type cohorts for each genotype also showed similar differences in fat and lean mass upon TRF. In addition, histological examination of the livers confirmed reduced lipids accumulation in TRF groups. Moreover, this feeding pattern protected the animals from rise in the serum TG and cholesterol, and relieved the insulin resistance condition noted in the ad Libitum groups.

Therefore, in summary, when the circadian clock mutant mice were placed on TRF regimen (with non-restricted total caloric intake), their usual characteristics of obesity and other metabolic problems (dyslipidemia, and insulin resistance) were largely relieved (3).

\section{Gut flora mediates the crosstalk between diet, circadian clock and health status}

While the gut microbiota is reported to have an important impact on our health, it also appears to have a reciprocal relationship with the circadian rhythm and eating habits (28). Indeed, ingestion of food was found to alter the inherent diurnal rhythm of gut microbiome, with both the contents of food, and timing of feeding playing a role in this process $(16,28,36)$. Furthermore, an earlier study pointed out that IF promoted white adipose browning and decreased obesity by shaping the gut flora (4). Another study showed that TRF decreased abundances of several obesogenic microbes, and increased the portion of presumed obesity-protective bacteria (16). Moreover, the intestinal flora appeared to interact with host clock genes (36), and regulate the host's circadian rhythm (37). An example was noted in a study which demonstrated that Enterobacter aerogenes contains an endogenous circadian clock that may synchronize with its host via melatonin secreted into the gut (38). Another study showed that circadian gene expression in the liver and intestine are likely to be affected by microbial products including unconjugated bile acids (39). In addition, it was observed that similar to the clock gene knockout mice, antibiotic-induced microbiota depletion in mice led to a prediabetic state with elevated glucose, free fatty acid, and triglyceride levels, which indicated that the gut microbiota plays a vital role in moderating the intestinal epithelial cells (IEC) homeostasis in these mice (40). In this study, the transcript profiles of 42 nuclear receptors (NRs) were examined in the ileum and colon of control and antibiotic-induced microbiotadepleted (AIMD) mice. Results showed that upon antibiotic treatment, transcripts of RevErb $\alpha$, PPAR $\alpha$, and CAR increased, and those of ROR $\alpha, E R \beta$, and PPAR $\beta$ decreased. Keeping in mind that ROR $\alpha$ and RevErb $\alpha$ belong to the clock machinery, expression of clock components in AIMD IEC was examined, and clock core was found to be disrupted too, so that Bmal1 and Cry1 transcripts decreased, whereas Per1 and Per2 transcripts increased. Further analysis showed that lack of microbiota resulted in corticosterone overproduction in ileal IEC. Usually the circadian corticosterone synthesis is cophasic in the ileal IEC and the adrenal gland, and they are independently regulated with ROR $\alpha$ and RevErb $\alpha$ playing key roles in IEC rhythmicity by inducing circadian expression of Tolllike receptors (TLRs) which recognize commensal bacterial products, and hence mediate microbiota signaling that control corticosterone synthesis via controlling PPAR $\alpha$ expression. which in turn is involved in corticosterone synthesis. In ileal IEC of AIMD mice, this mechanism is missing, leading to corticosterone overproduction, resulting in dysregulation of glucose and lipid homeostasis (40).

From the above, it can be concluded that a healthy gut flora is required for the maintenance of metabolic homeostasis, and over all health status of the host. It is also important to note that cyclical alteration of the gut flora in response to the feeding/fasting rhythms contribute to the diversity and function of microbiota $(16,41,42)$. Moreover, the peak-totrough differences of gut microbiome caused by feeding/ fasting rhythms allow the robustness of circadian clock, while frequent eating could dampen molecular circadian clock, and cause chronic disruption of our overall homeostasis (6).

Finally it is worth noting that unlike the adult population, pediatric mice showed severe irreversible unfavorable outcomes under TRF (43). The underlying logic lies in the completely different physiology of children and adults. Therefore, the best feeding regimen should follow the rule 
of 'Let nature take its course'.

In conclusion, the benefits of IF and TRF are not simple translation of the fasting physiology, but instead they represent complex interactions between rhythmic digestion of food, gut microbiome, and circadian clock. Further investigations are called for to acquire a holistic understanding of this highly sophisticated molecular blueprint. This might help to develop well designed diet therapy that can control various chronic ill health conditions and diseases. Furthermore, it could guide the development and testing of pharmacologic interventions that mimic the health and disease-modifying benefits of IF for those who are not willing, or able to carry this type of feeding regimen

\section{Acknowledgments}

Funding: None.

\section{Footnote}

Reporting Checklist: The authors have completed the Narrative Review reporting checklist. Available at http:// dx.doi.org/10.21037/hbsn-20-317

Conflicts of Interest: All authors have completed the ICMJE uniform disclosure form (available at http://dx.doi. org/10.21037/hbsn-20-317). DH serves as an unpaid editorial board member of Hepatobiliary Surgery and Nutrition. The other authors have no conflicts of interest to declare.

Ethical Statement: The authors are accountable for all aspects of the work in ensuring that questions related to the accuracy or integrity of any part of the work are appropriately investigated and resolved.

Open Access Statement: This is an Open Access article distributed in accordance with the Creative Commons Attribution-NonCommercial-NoDerivs 4.0 International License (CC BY-NC-ND 4.0), which permits the noncommercial replication and distribution of the article with the strict proviso that no changes or edits are made and the original work is properly cited (including links to both the formal publication through the relevant DOI and the license). See: https://creativecommons.org/licenses/by-nc-nd/4.0/.

\section{References}

1. Weindruch R, Sohal RS. Seminars in medicine of the Beth
Israel Deaconess Medical Center. Caloric intake and aging. N Engl J Med 1997;337:986-94.

2. Mager DE, Wan R, Brown M, et al. Caloric restriction and intermittent fasting alter spectral measures of heart rate and blood pressure variability in rats. FASEB J 2006;20:631-7.

3. Chaix A, Lin T, Le HD, et al. Time-Restricted Feeding Prevents Obesity and Metabolic Syndrome in Mice Lacking a Circadian Clock. Cell Metab 2019;29:303-19.e4.

4. Li G, Xie C, Lu S, et al. Intermittent Fasting Promotes White Adipose Browning and Decreases Obesity by Shaping the Gut Microbiota. Cell Metab 2017;26:672-85.e4.

5. Fontán-Lozano Á, Sáez-Cassanelli JL, Inda MC, et al. Caloric restriction increases learning consolidation and facilitates synaptic plasticity through mechanisms dependent on NR2B subunits of the NMDA receptor. J Neurosci 2007;27:10185-95.

6. Hatori M, Vollmers C, Zarrinpar A, et al. Timerestricted feeding without reducing caloric intake prevents metabolic diseases in mice fed a high-fat diet. Cell Metab 2012;15:848-60.

7. Mattson MP, Allison DB, Fontana L, et al. Meal frequency and timing in health and disease. Proc Natl Acad Sci U S A 2014;111:16647-53.

8. Barnosky AR, Hoddy KK, Unterman TG, et al. Intermittent fasting vs daily calorie restriction for type 2 diabetes prevention: A review of human findings. Transl Res 2014;164:302-11.

9. Gill S, Le HD, Melkani GC, Panda S. Time-restricted feeding attenuates age-related cardiac decline in Drosophila. Science 2015;347:1265-9.

10. Chung H, Chou W, Sears DD, et al. Time-restricted feeding improves insulin resistance and hepatic steatosis in a mouse model of postmenopausal obesity. Metabolism 2016;65:1743-54.

11. Longo VD, Panda S. Fasting, Circadian Rhythms, and Time-Restricted Feeding in Healthy Lifespan. Cell Metab 2016;23:1048-59.

12. Mattson MP, Longo VD, Harvie M. Impact of intermittent fasting on health and disease processes. Ageing Res Rev 2017;39:46-58.

13. De Cabo R, Mattson MP. Effects of intermittent fasting on health, aging, and disease. N Engl J Med 2019;381:2541-51.

14. Ren J, Hu D, Mao Y, et al. Alteration in gut microbiota caused by time-restricted feeding alleviate hepatic ischaemia reperfusion injury in mice. J Cell Mol Med 2019;23:1714-22. 
15. Chapnik N, Genzer Y, Froy O. Relationship between FGF21 and UCP1 levels under time-restricted feeding and high-fat diet. J Nutr Biochem 2017;40:116-21.

16. Zarrinpar A, Chaix A, Yooseph S, et al. Diet and feeding pattern affect the diurnal dynamics of the gut microbiome. Cell Metab 2014;20:1006-17.

17. Mattson MP, Moehl K, Ghena N, et al. Intermittent metabolic switching, neuroplasticity and brain health. Nat Rev Neurosci 2018;19:63-80.

18. Inoki K, Kim J, Guan KL. AMPK and mTOR in Cellular Energy Homeostasis and Drug Targets. Annu Rev Pharmacol Toxicol 2012;52:381-400.

19. Panda S. Circadian physiology of metabolism. Science 2016;354:1008-15.

20. Mattson MP. An Evolutionary Perspective on Why Food Overconsumption Impairs Cognition. Trends Cogn Sci 2019;23:200-12.

21. Hardin PE, Panda S. Circadian timekeeping and output mechanisms in animals. Curr Opin Neurobiol 2013;23:724-31.

22. Ueda HR, Chen W, Adachi A, et al. A transcription factor response element for gene expression during circadian night. Nature 2002;418:534-9.

23. Cho H, Zhao X, Hatori M, et al. Regulation of circadian behaviour and metabolism by REV-ERB- $\alpha$ and REVERB- $\beta$. Nature 2012;485:123-7.

24. Sato TK, Panda S, Miraglia LJ, et al. A functional genomics strategy reveals rora as a component of the mammalian circadian clock. Neuron 2004;43:527-37.

25. Bugge A, Feng D, Everett LJ, et al. Rev-erb $\alpha$ and Reverb $\beta$ coordinately protect the circadian clock and normal metabolic function. Genes Dev 2012;26:657-67.

26. Feng D, Liu T, Sun Z, et al. A circadian rhythm orchestrated by histone deacetylase 3 controls hepatic lipid metabolism. Science 2011;331:1315-9.

27. Koike N, Yoo SH, Huang HC, et al. Transcriptional architecture and chromatin landscape of the core circadian clock in mammals. Science 2012;338:349-54.

28. Thaiss CA, Zeevi D, Levy M, et al. Transkingdom control of microbiota diurnal oscillations promotes metabolic homeostasis. Cell 2014;159:514-29.

29. Oike H, Nagai K, Fukushima T, Ishida N, Kobori $M$. Feeding cues and injected nutrients induce acute expression of multiple clock genes in the mouse liver. PLoS One 2011;6:e23709.

30. Yasumoto Y, Hashimoto C, Nakao R, et al. Short-term feeding at the wrong time is sufficient to desynchronize peripheral clocks and induce obesity with hyperphagia, physical inactivity and metabolic disorders in mice.
Metabolism 2016;65:714-27.

31. Zheng X, Sehgal A. AKT and TOR signaling set the pace of the circadian pacemaker. Curr Biol 2010;20:1203-8.

32. Lamia KA, Sachdeva UM, Di Tacchio L, et al. AMPK regulates the circadian clock by cryptochrome phosphorylation and degradation. Science 2009;326:437-40.

33. Asher G, Gatfield D, Stratmann M, et al. SIRT1 Regulates Circadian Clock Gene Expression through PER2 Deacetylation. Cell 2008;134:317-28.

34. Masri S, Sassone-Corsi P. The circadian clock: A framework linking metabolism, epigenetics and neuronal function. Nat Rev Neurosci 2013;14:69-75.

35. Kohsaka A, Laposky AD, Ramsey KM, et al. HighFat Diet Disrupts Behavioral and Molecular Circadian Rhythms in Mice. Cell Metab 2007;6:414-21.

36. Heath-Heckman EAC, Peyer SM, Whistler CA, et al. Bacterial bioluminescence regulates expression of a host cryptochrome gene in the squid-vibrio symbiosis. mBio 2013;4:e00167-13.

37. Parkar SG, Kalsbeek A, Cheeseman JF. Potential role for the gut microbiota in modulating host circadian rhythms and metabolic health. Microorganisms 2019;7:41.

38. Paulose JK, Cassone VM. The melatonin-sensitive circadian clock of the enteric bacterium Enterobacter aerogenes. Gut Microbes 2016;7:424-7.

39. Govindarajan K, MacSharry J, Casey PG, et al. Unconjugated bile acids influence expression of circadian genes: A potential mechanism for microbe-host crosstalk. PLoS One 2016;11:e0167319.

40. Mukherji A, Kobiita A, Ye T, et al. Homeostasis in intestinal epithelium is orchestrated by the circadian clock and microbiota cues transduced by TLRs. Cell 2013;153:812-27.

41. Voigt RM, Forsyth CB, Green SJ, et al. Circadian Rhythm and the Gut Microbiome. Int Rev Neurobiol 2016;131:193-205.

42. Hu D, Ye Y, Mao Y, et al. Time-restricted feeding during childhood has persistent effects on mice commensal microbiota. Ann Transl Med 2019;7:556.

43. Hu D, Mao Y, Xu G, et al. Time-restricted feeding causes irreversible metabolic disorders and gut microbiota shift in pediatric mice. Pediatr Res 2019;85:518-26.

Cite this article as: $\mathrm{Hu} \mathrm{D,} \mathrm{Xie} \mathrm{Z,} \mathrm{Ye} \mathrm{Y,} \mathrm{Bahijri} \mathrm{S,} \mathrm{Chen} \mathrm{M.}$ The beneficial effects of intermittent fasting: an update on mechanism, and the role of circadian rhythm and gut microbiota. HepatoBiliary Surg Nutr 2020;9(5):597-602. doi: 10.21037/hbsn-20-317 